\title{
Sistema de Gerenciamento de um Estacionamento Inteligente utilizando sensores de efeito Hall
}

\author{
Givanildo Lima do Nascimento Júnior ${ }^{1}$, Valdir de Souza Junior ${ }^{1}$, André Luiz de \\ Oliveira Cezário ${ }^{1}$, José Estevam Vilar Borges ${ }^{1,2}$
}

${ }^{1}$ Instituto de Computação - Universidade Federal de Alagoas (UFAL)

Cep: 57072-970 - Maceió - AL - Brasil

${ }^{2}$ Centro Universitário Mario Pontes Jucá - UMJ

Cep: 57040-600 - Maceió - AL - Brasil

\{glnjunior, vsj, aloc, estevam\}@ic.ufal.br

\begin{abstract}
This research aims to present an automated solution that streamlines search for parking spaces, in public or private parking lots. In order to accelerate this process, this article proposes a didactic system inserted in the Arduino platform integrated with Hall effect sensors responsible for identifying the vehicles in each spot. The results obtained with the prototype indicate the economic feasibility of expanding this project to a real environment, given the low cost of the components used, indicated at work, making the experience more comfortable for whoever's using the system.
\end{abstract}

Resumo. Esta pesquisa tem por finalidade apresentar uma solução automatizada que agilize a busca por vagas, em estacionamentos públicos ou privados. Visando acelerar este processo, é proposto neste artigo um sistema didático inserido em plataforma Arduino integrado a sensores de efeito Hall responsáveis por identificar os veículos em cada vaga. Os resultados obtidos com o protótipo resultante indicam a viabilidade econômica de expansão deste projeto para um ambiente real, visto o baixo custo dos componentes utilizados, indicado no trabalho, proporcionando mais conforto ao cliente que fizer uso do sistema.

\section{Introdução}

A crescente necessidade de veículos automotivos para locomoção de pessoas em função da precariedade do transporte público e o aumento significativo da frota automobilística é uma realidade nas grandes cidades brasileiras, segundo dados Associação Nacional dos Detrans (AND). Com o aumento da frota, o Brasil já tem um automóvel a cada 4,4 habitantes. No infográfico em [Reis 2019], podemos conhecer melhor a distribuição da frota pelo país, além de entender como essa frota se divide em cada região entre carros e motocicletas.

Nesse sentido, disponibilizar vagas e garantir estacionamento rápido, seguro e sem aborrecimentos é um desafio permanente para clientes e empresários [Napoli, Nocera e Rossi 2014], visto que, é amplamente reconhecido que os motoristas que procuram estacionar em grandes áreas urbanas desperdiçam tempo e combustível, aumentando assim o congestionamento do tráfego e a poluição do ar [Polycarpou, Lambrinos e Protopapadakis 2013]. 
A automação de estacionamentos proporciona melhor aproveitamento das vagas existentes, ordena o fluxo e a circulação de veículos, além de fornecer uma completa informação gerencial para o controle eficaz do uso das vagas e da receita obtida pela sua ocupação [Leandro 2012]. Avanços e inovações na área tecnológica, como a comunicação sem fio e os dispositivos móveis, criaram a possibilidade de se transferir serviços de computadores convencionais para ambientes próximos ao ser humano, utilizando sistemas embarcados [Manogna e Dakannagari 2016].

Neste trabalho é apresentado um sistema que busca automatizar por completo um estacionamento, de modo a garantir que os usuários possam usufruir de todas as comodidades proporcionadas por um estacionamento inteligente.

O sistema proposto consiste em uma solução automatizada que gerencia o número de vagas disponíveis, bem como o controle de entrada de veículos em um estacionamento. $\mathrm{O}$ sistema foi implementado numa maquete, cuja representação em escala reduzida simula um estacionamento com quatro vagas. Há uma cancela para entrada e outra para saída, além de um display indicando o número de vagas disponíveis. Caso ainda hajam vagas a cancela abre automaticamente com a chegada do veículo e fecha após 5 segundos, permitindo que o veículo passe tranquilamente. Esse valor foi adotado dado que está sendo utilizada uma maquete, sendo facilmente adaptável em caso de uso em uma sistema real. Além disso, o sistema possui um conjunto de leds RGB na entrada, sendo um correspondente a cada umas das 4 vagas existentes no estacionamento, que indicam se determinada vaga está livre ou não. Caso o led de determinada vaga esteja verde, isso indica que a vaga está livre, enquanto que se o led estiver vermelho, a vaga já está ocupada. Dessa forma, o usuário do sistema, além de saber o número de vagas disponíveis, recebe uma indicação de quais as vagas que estão livres, proporcionando uma redução considerável do tempo de procura, evitando eventuais congestionamentos e garantindo economia de combustível.

A principal contribuição deste trabalho é desenvolver um sistema de estacionamento inteligente onde o uso dos sensores de efeito Hall impeça que outros objetos que não os veículos sejam detectados de modo que a vaga seja dada como livre, diferente do que aconteceria, por exemplo, no caso de um sensor infravermelho, que detectaria um carrinho de compras em um estacionamento de supermercado. Como o custos dos sensores de efeito Hall e o infravermelho é o mesmo (o custo de ambos no início do desenvolvimento deste trabalho era de $\mathrm{R} \$ 9,90$ ), então a troca não implica custos adicionais ao desenvolvimento do projeto e ainda assim, a escolha do sensor de efeito Hall previne problemas como o já mencionado.

Vale destacar que não foi implementado um módulo de reserva de vagas à distância porque a abordagem foi restrita a um ambiente em que esse tipo de situação não faria sentido, como supermercados, shoppings e afins.

\section{Trabalhos Relacionados}

Em decorrência dos diversos aspectos abordados da introdução deste trabalho, diversos estudos têm sido desenvolvidos a fim de desenvolver os chamados smart parks, com diversas abordagens distintas.

Em [Vicente 2016] é apresentado um sistema com uma camada de sensoriamento, composta por sensores ultrassônicos associados à um microcontrolador, os quais tinham a tarefa de fazer a verificação das vagas e enviar os dados para a 
nuvem. Além disso, há uma camada de aplicação que possui uma aplicação web que permite a interação com o usuário e permite que ele possa reservar sua vaga à distância.

Em [Thangam 2018] é proposto um sistema de reservas para um smart parking utilizando o reconhecimento óptico de caracteres e o reconhecimento facial para fornecer segurança com um Raspberry Pi.

Em [Silva 2013], por sua vez, é projetado um sistema que permite fazer uma comunicação sem fio entre a vaga do estacionamento e uma central de controle, de onde se monitora todo o sistema. A comunicação entre os dois sistemas consiste na interação de dispositivos diferentes, onde o sistema localizado na vaga se dá por meio da interligação entre um sensor ultrassônico, a plataforma microcontrolada Arduino e o um rádio emissor, que utiliza a tecnologia de comunicação sem fio Zigbee.

Com relação aos trabalhos acima citados, este projeto possui como principal vantagem sua facilidade de implementação e manutenção, visto que o projeto apresenta uma solução mais simplória para o problema de automatização de um estacionamento, embora ainda seja muito efetiva e funcional para as tarefas propostas.

\section{Metodologia}

A metodologia adotada neste trabalho consiste na composição de diversas etapas, as quais foram testadas e implementadas de forma gradativa para melhor análise de erros e organização. Para cada implementação foi necessária a análise dos componentes que integram o protótipo de um estacionamento inteligente que utiliza sensores de efeito Hall, além de uma plataforma de prototipagem eletrônica Arduino.

Nas seções a seguir é definido o efeito hall e são descritos o funcionamento do sistema, materiais utilizados e como foi dado o desenvolvimento das etapas de hardware e software do sistema, explicitando como ocorre a integração entre as partes.

\subsection{O Efeito Hall}

Os sensores de campos magnéticos são dispositivos importantes em um grande número de aplicações que vão desde aparelhos de consumo até máquinas industriais.

O Efeito Hall é observado quando uma placa metálica ou semicondutora, na qual flui uma corrente elétrica, é inserida em um campo magnético no qual as linhas de fluxo são perpendiculares à placa e à corrente. O efeito consiste na deflexão da corrente, gerando uma diferença de potencial segundo uma direção normal a ela. Esta diferença de potencial é diretamente proporcional à intensidade do campo magnético e da corrente elétrica [Kirst 2006].

Conforme descrito por [Patsko 2006] quando uma corrente passa por um material condutor, ela é distribuída uniformemente ao longo do material e não há nenhuma diferença de potencial entre as laterais do condutor. Entretanto, ao aproximar um imã, o campo magnético perturbará a distribuição da corrente ao longo do material, havendo um acúmulo de cargas negativas em um dos lados do condutor, gerando uma 
tensão entre suas laterais. Se o sentido do campo magnético for invertido, a tensão presente no material também será invertida, como pode ser visto na Figura 1.

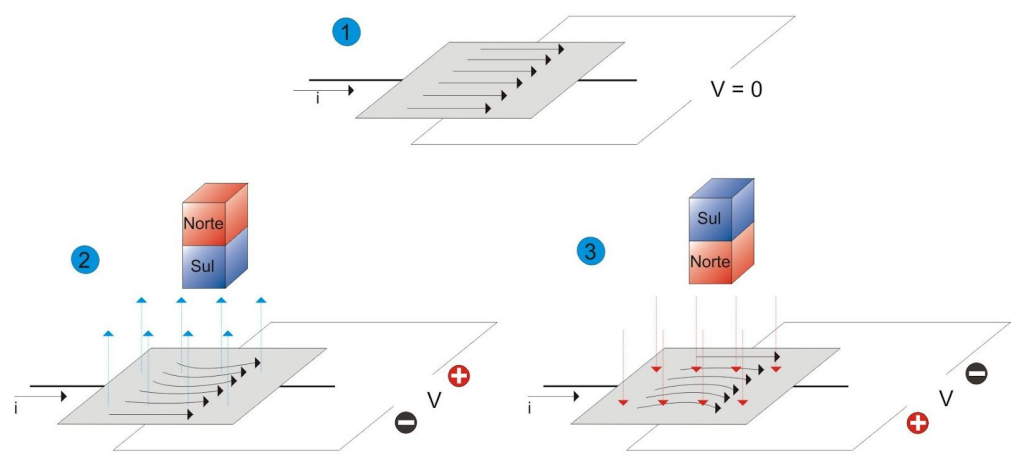

Figura 1. llustração do efeito Hall

Essa diferença de tensão é pequena, mas se for amplificada, pode ser monitorada por um circuito externo, o que é feito internamente pelo próprio sensor. A saída do sensor pode ser analógica ou digital. Sensores analógicos possuem uma saída correspondente à intensidade do campo magnético que incide sobre eles. Já nos digitais, sua saída estará num nível lógico alto enquanto não houver um campo magnético. Se o campo magnético ultrapassar certo valor, então a sua saída se encontrará num nível lógico baixo [Patsko 206].

\subsection{Funcionamento}

O funcionamento do sistema é dado a partir da análise contínua da disponibilidade de vagas, através do uso de sensores de efeito Hall (Hall KY-003) em cada uma das 4 vagas do estacionamento.

Em cada entrada foi utilizado um servomotor (Micro Servo 9g SG90 TowerPro) acoplando-se um suporte às hélices que acompanham o dispositivo para simular a abertura e fechamento da cancela.

Esse controle de entrada é dado de acordo com número de vagas, sinalizado em um display. Se ainda houverem vagas disponíveis, um sensor de efeito Hall identifica a chegada de um novo veículo e abre a cancela, que posteriormente é fechada com a passagem do veículo. Caso todas as vagas estejam ocupadas, isto também é sinalizado no display, porém a cancela não é aberta.

Além disso, serão utilizados 4 leds RGB para sinalizar as vagas que estão livres, facilitando a identificação por parte do condutor. Há um para cada vaga e a indicação é feita a partir da cor: leds verdes indicam que sua vaga correspondente está livre, enquanto a cor vermelha indica que a vaga correspondente está ocupada. Na Figura 2 é ilustrado um esboço do sistema. 


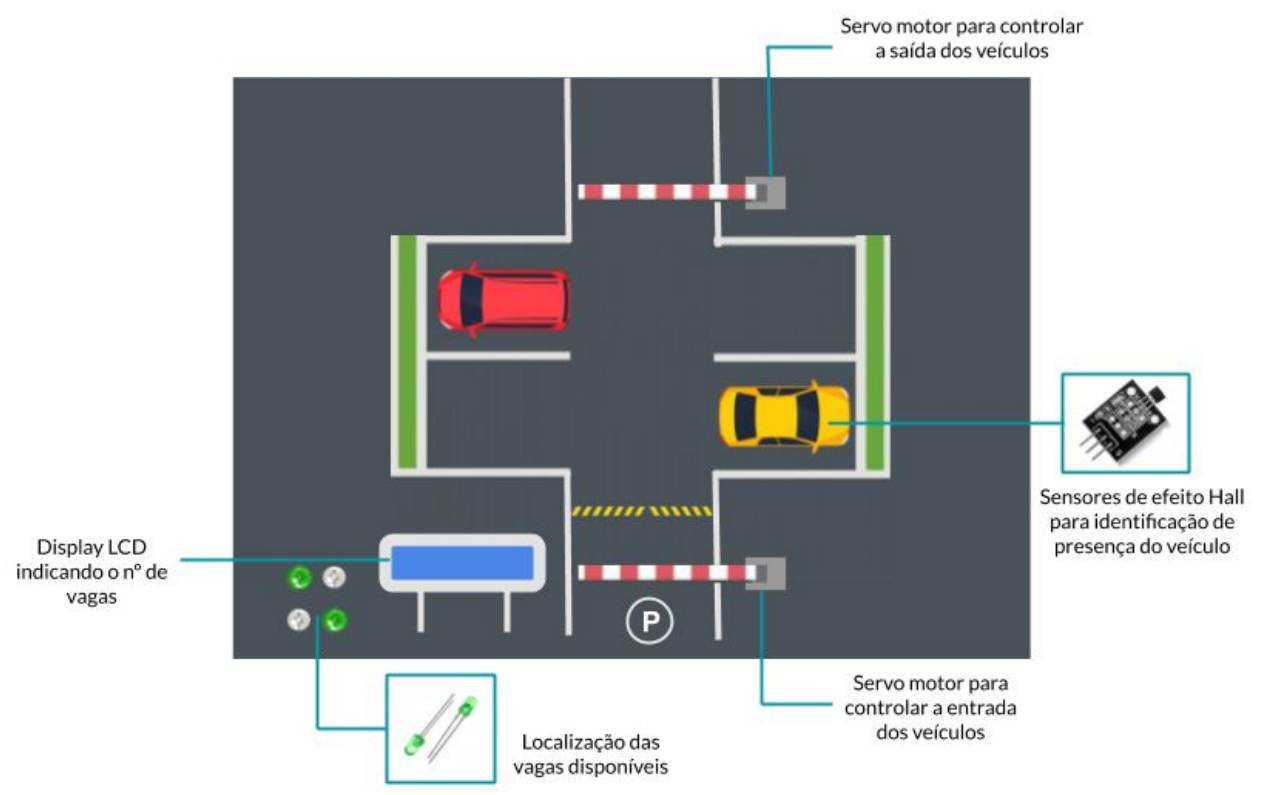

Figura 2. Esquema do sistema de estacionamento proposto

Na tabela 1, são listados todos os equipamentos componentes da maquete do sistema desenvolvido, com as respectivas quantidades utilizadas.

Tabela 1. Lista de Equipamentos utilizados

\begin{tabular}{|c|c|c|c|}
\hline Item & Nome & Quantidade & Custo Total \\
\hline 1 & Sensor Hall KY-003 & 6 & $\mathrm{R} \$ 59,40$ \\
\hline 2 & $\begin{array}{l}\text { Micro Servo 9g SG90 } \\
\text { TowerPro }\end{array}$ & 2 & $\mathrm{R} \$ 35,80$ \\
\hline 3 & Arduino Mega & 1 & $\mathrm{R} \$ 109,90$ \\
\hline 4 & $\begin{array}{c}\text { Display LCD } 16 \times 2 \text { Backlight } \\
\text { Verde }\end{array}$ & 1 & $\mathrm{R} \$ 24,90$ \\
\hline 5 & Led RGB & 4 & $\mathrm{R} \$ 3,96$ \\
\hline 6 & Ímã Ferrite Disco 20x6 mm & 40 & $\mathrm{R} \$ 4,20$ \\
\hline 7 & $\begin{array}{c}\text { Kit de Resistores } 470 \Omega 1 / 4 \mathrm{~W} \\
\text { com } 20 \text { unidades }\end{array}$ & 1 & $\mathrm{R} \$ 2,90$ \\
\hline 8 & $\begin{array}{c}\text { Kit de Jumpers com } 65 \\
\text { unidades }\end{array}$ & 1 & $\mathrm{R} \$ 14,90$ \\
\hline 9 & $\begin{array}{c}\text { Foam board } \\
70 \mathrm{~cm} \times 50 \mathrm{~cm} \times 5 \mathrm{~mm}\end{array}$ & 1 & $\mathrm{R} \$ 20,40$ \\
\hline \multicolumn{3}{|c|}{ Custo Total } & $\mathrm{R} \$ 276,36$ \\
\hline
\end{tabular}




\subsection{Circuito}

Quanto a montagem do circuito, adotamos a padronização de VCC como 5V para todos os componentes, que é um valor dentro da faixa de operação de todos os componentes. Além disso, considerando ter o máximo de eficiência energética, escolhemos uma tensão única para evitar o uso de transformadores de tensão e assim, conforme [Say 1983], evitar perdas de correntes magnetizantes e também de correntes de fuga decorrentes do uso do transformador.

Utilizamos uma configuração básica para acionamento dos servo-motores, consistindo de um diodo para prevenir correntes no sentido contrário que podem danificar o restante do circuito. Os sensores estão ligados às portas analógicas do Arduino e os servo-motores são acionados por meio de portas PWM, onde seu ângulo é variado em até 90 graus representando a abertura e fechamento das cancelas.

\subsection{Programação Arduino}

Para utilização dos sensores, o efeito Hall é linear e cresce proporcionalmente com a intensidade do campo magnético, portanto foi possível fazer a leitura dos sensores utilizando a entrada analógica do Arduino, onde foi adotado um limiar indicando as faixas na qual há a presença do campo magnético dos carrinhos.

Para compreender o funcionamento do sensor, foram realizadas várias leituras de tensão consecutivas, variando a distância de um ímã ao sensor. As leituras obtidas são apresentadas na figura 3. Como a leitura ociosa média dos sensores sem a presença do ímã girava em torno de $2,1 \mathrm{~V}$, buscamos uma distância em que a leitura fosse ao menos superior a $2,5 \mathrm{~V}$, o que ocorreu quando o ímã estava a, no máximo, $1,9 \mathrm{~cm}$ de distância do sensor (ponto marcado com um "x" em vermelho na Figura 3), onde a leitura de tensão foi de $2,65 \mathrm{~V}$. Dessa forma, diz-se que há um veículo na vaga quando a leitura proveniente do sensor é de ao menos $2,65 \mathrm{~V}$, o que ocorre a partir de $1,9 \mathrm{~cm}$ de distância. Embora pareça pequena, a distância é coerente com a escala dos itens utilizados no protótipo.

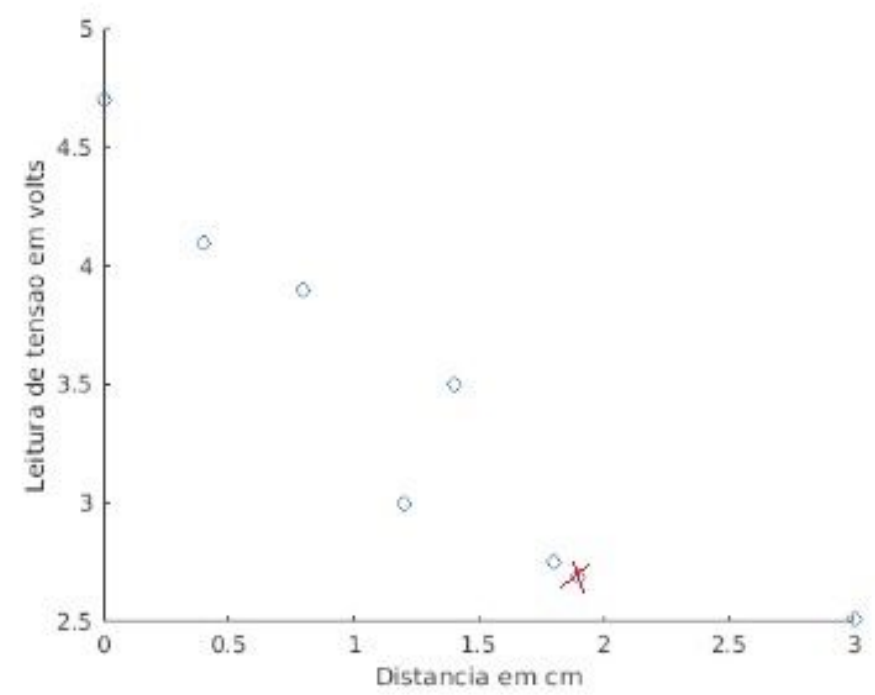

Figura 3. Tensão x Distância com sensores de efeito Hall 
O sistema foi dividido em duas etapas, o despertar e o funcionamento, propriamente dito. Ao iniciar, os sensores efetuam a leitura de 10 amostras com um intervalo de 1 milissegundo cada, sendo calculada uma média dessas amostras. Todas essas médias são salvas para futura comparação com o limiar, visto que elas representam o estado do sensor sem a presença de carros, isto é, sua leitura "ociosa". Ao final, é determinado uma faixa de valores que representam o estado "ocioso" comum de todos os sensores utilizados.

$\mathrm{Na}$ seção de funcionamento, é utilizada uma aproximação similar a implementação de um filtro passa-baixa à nível de código, na qual são efetuadas 10 leituras em cada sensor e então é calculada uma média das leituras. Com isso, espera-se que o ruído de alta frequência seja removido.

A nível de projeto, o controle do número de vagas é feito exclusivamente pelos sensores da entrada e saída e não pelos sensores das vagas de estacionamento. Essa escolha foi importante para evitar o atraso entre a entrada de veículos e seu posicionamento perante o sensor, pois se os sensores forem utilizados na contabilização de vagas, isso pode implicar em uma situação na qual ocorre a entrada de mais carros do que o permitido, visto o número de vagas no local. Ou seja, como a contagem é feita nos sensores das cancelas, a quantidade de vagas só é atualizada após a saída de um veículo do estacionamento e não assim que ele sai da vaga que estava ocupando.

\section{Resultados}

Para facilitar a visualização do funcionamento do sistema, foi construída uma maquete para simular um ambiente real de aplicação, como mostra a Figura 4. Foram utilizados carrinhos em miniatura e a cada um deles foram presos ao menos 8 ímãs, de modo a gerar um campo eletromagnético em cada carrinho que é captado pelos sensores de efeito Hall caso os carrinhos estejam próximos.

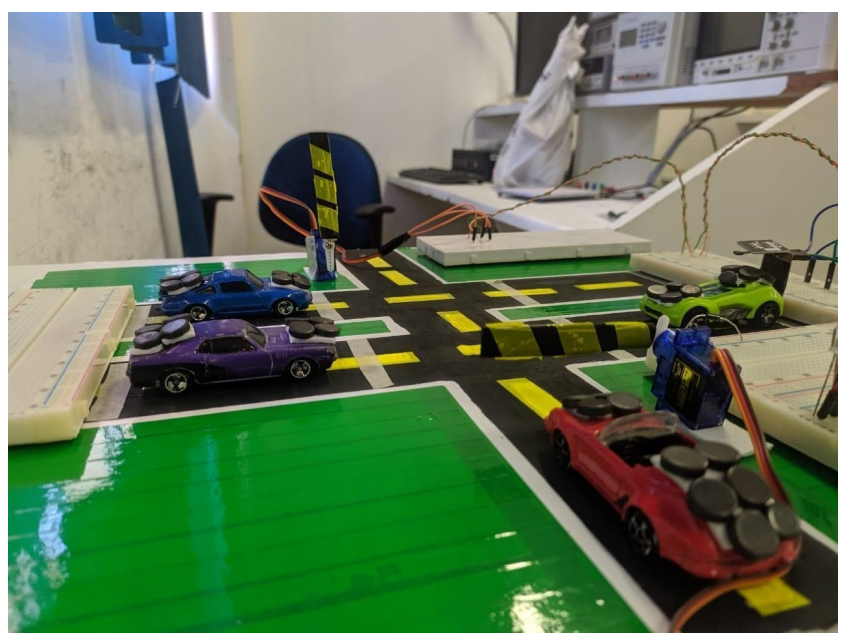

Figura 4. Maquete do Estacionamento

Os sensores foram presos em um modelo de poste, sendo estes previamente confeccionados, atrás de cada uma das vagas, como mostra a Figura 5, de modo que a leitura do sensor indica presença ou não de um carrinho a ocupando.

A leitura de cada um desses sensores é utilizada como referência para indicar se determinada vaga está ocupada ou não e, a partir disso, essa indicação é utilizada para 
indicar a cor a ser adotada para o led de cada vaga, sendo vermelho para caso ela esteja ocupada e verde para caso ela esteja livre.

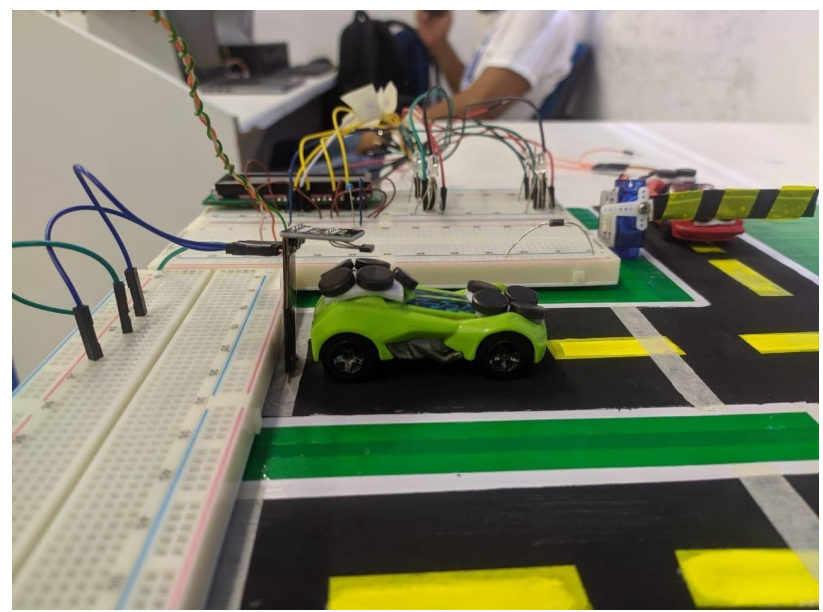

Figura 5. Poste com Sensor Hall

Em todos os testes realizados, os resultados obtidos foram considerados bastante satisfatórios, dado que o sistema respondeu muito bem as simulações. O tempo adotado de 5 seg para fechamento da cancela foi suficiente para passagem do veículo, sendo algo facilmente adaptável caso fosse necessário modificar. Além disso, todos os sensores indicaram corretamente a presença dos veículos nas vagas, ainda que não estivessem tão bem estacionados e os leds RGB, consequentemente, responderam corretamente com relação ao estado de cada vaga; a contagem de vagas disponíveis e apresentação no display, gerenciada pelo sensores presentes nas cancelas, funcionou de forma esperada, bloqueando a passagem de novos veículos quando o estacionamento já se encontrava com lotação máxima.

\section{Conclusão}

Este trabalho apresenta um sistema de estacionamento inteligente que funciona sem a necessidade de interferência humana, visando colaborar com uma solução de baixo custo para o problema da ausência de vagas para estacionar proveniente do aumento da frota de veículos nas cidades brasileiras.

Através do direcionamento das vagas e do controle da entrada de veículos, o tempo gasto com a procura de um local para estacionar é minimizado, o que implica na diminuição do intervalo de tempo que o veículo permanece ligado e consequentemente promove economia de custos com combustível e reduz a emissão de gases na atmosfera. Os resultados obtidos nos testes foram considerados satisfatórios, visto que todo o sistema respondeu corretamente as tarefas, mostrando que as constantes adotadas para o tempo de movimentação da cancela e indicação da presença de veículo na vaga por leitura de tensão atenderam os requisitos, comprovando a eficácia do sistema de estacionamento inteligente. 
Em nossos trabalhos futuros, pretendemos realizar uma ampliação do sistema, aumentando o número de andares do projeto, além de incluir vagas especiais (para idosos e/ou grávidas) com suas respectivas restrições e indicação diferenciada.

\section{Agradecimentos}

Os autores agradecem ao Instituto de Computação da UFAL, por disponibilizar o espaço e parte do material utilizado na confecção deste projeto, além de todos que, de alguma forma, contribuíram para sua execução.

\section{Referências}

REIS, Thiago; ARAGÃO, Leo. Frota de carros e motos no país. G1, 2013. Disponível em: <http://g1.globo.com/carros/frota-carros-motos-2013/>. Acesso em: 05 de ago. 2019.

Di Napoli, C., Di Nocera, D. and Rossi, S., 2014. Negotiating parking spaces in smart cities. In Proceeding of the 8th International Workshop on Agents in Traffic and Transportation, in conjunction with AAMAS.

Polycarpou, E., Lambrinos, L. and Protopapadakis, E., 2013, June. Smart parking solutions for urban areas. In 2013 IEEE 14th International Symposium on" A World of Wireless, Mobile and Multimedia Networks"(WoWMoM) (pp. 1-6). IEEE.

V. L. S. Leandro, Projeto Vaga Fácil: Uma solução automatizada na viabilização de vagas em estacionamentos públicos e privados. ISBN 978-85-62830-10-5. VII CONNEPI, 2012.

Manogna, S., Dakannagari, H.R. (2016). "Internet of Things". In International Journal of Computer Science and Information Technologies, p. 1567-1570. 2016.

Vicente, T.P.R., Controle Inteligente de Vagas para Estacionamentos Utilizando o Conceito de Internet das Coisas. 2016.

Thangam, E.C., Mohan, M., Ganesh, J. and Sukesh, C.V., 2018. Internet of Things (IoT) based Smart Parking Reservation System using Raspberry-pi. International Journal of Applied Engineering Research, 13(8), pp.5759-5765.

Silva, A. D. R; et al. Prototipagem de estacionamento inteligente com comunicação sem Fio. Mostra Nacional de Robótica (MNR) 2013.

Kirst, R. W.; et al. Desenvolvimento de sensores de efeito hall para utilização em ensaios triaxiais com sucção controlada.Salão de iniciação Científica (18. : 2006 : Porto Alegre, RS). Livro de resumos. Porto Alegre : UFRGS, 2006.

Patsko, L. F.; Tutorial Aplicações, Funcionamento e Utilização de Sensores, 2006.

Say, M. G. (1983). Alternating Current Machines (5th ed.). London: Pitman. ISBN 978-0-273-01969-5. 